\title{
Tissue Culture Studies on Fortunella polyandra 'Nagami' and 'Meiwa'
}

\author{
N. Mohamed, R.M. Taha, H. Elias, S. Abdullah and N.A. Hasbullah \\ Institute of Biological Sciences \\ Faculty of Sciences \\ University of Malaya \\ 50603 Kuala Lumpur \\ Malaysia
}

Keywords: BAP, callus, explants, Fortunella polyandra, MS medium, NAA, regeneration

\begin{abstract}
Studies of Fortunella polyandra 'Nagami' and 'Meiwa' were carried out to observe their responses in tissue culture systems. Explant sources investigated included roots, stems, leaves and cotyledons which were cultured on Murashige and Skoog (MS) medium supplemented with different combinations and concentrations of various hormones. The hormones used were naphthalene acetic acid (NAA) and benzylaminopurine (BAP) at $\mathrm{pH} 5.8$ with a temperature of $23-26^{\circ} \mathrm{C}$ and a photoperiod of 16 hours light and 8 hours dark. The pH of the media was also altered to include 4.8, 5.8, 6.8 and 7.8. The various explants were subjected to light and dark treatments in order to study the morphogenesis of this species. From the results, it was observed that stem and leaf explants were more responsive than other explants. The best media for regeneration and callus formation was MS supplemented with $0.5 \mathrm{mg} / \mathrm{L} \mathrm{NAA}$ and $0.5 \mathrm{mg} / \mathrm{L}$ BAP at $\mathrm{pH} 5.8$ for 'Nagami' and $\mathrm{pH} 7.8$ for 'Meiwa'. Regeneration was achieved via direct organogenesis and also via callus formation. The highest percentage of shoot formation $(40 \%)$ was obtained from stem explants ('Meiwa') cultured on MS supplemented with $0.5 \mathrm{mg} / \mathrm{L}$ NAA and $0.5 \mathrm{mg} / \mathrm{L}$ BAP at pH 7.8.
\end{abstract}

\section{INTRODUCTION}

Plant tissue culture involves culturing of explants on media containing hormones or plant growth regulators and nutrients to support the growth of explants. Plant tissue culture has many applications and offers many practical benefits. Tissue culture studies also help to identify cultivars that are resistant to pesticides and diseases. Fortunella polyandra, commonly known as Malayan Kumquat, related to Citrus in the flowering plant family Rutaceae. They are planted outdoors in pots or in the ground. They are thorny and do not possess an aromatic smell when crushed.

Tropical rare fruits are generally neglected because they have not been exploited commercially and there is a lack of improved cultivars. These fruit species also experience alternate or irregular fruiting seasons and have restricted habitats. In spite of these constraints, many of these species can be promoted or introduced to local consumers and overseas markets. This species consists of many cultivars with variety of shapes such as 'Nagami', 'Marumi', 'Meiwa' and 'Hong Kong' (Swingle, 1915).

'Nagami' and 'Meiwa' were chosen in this research. Oval kumquat or known as 'Nagami' is oval in shape. It has a relatively small number of segments (four to five) and contains 2 to 5 seeds. 'Nagami' fruit have a deep colour and a distinctive flavour and are in season from October to January. 'Meiwa' kumquat is a natural hybrid between the oval ('Nagami') and round ('Marumi') kumquats. The most distinctive features of this kumquat are the short oblong or round form and relatively large size of the fruit, the more numerous sections (commonly seven), comparatively sweet flavour, and low seed content. Many fruits are seedless. This is the best kumquat cultivar for eating fresh. Although slightly less cold-hardy than 'Nagami' it is increasing in popularity. They have been used as candying and kumquat preserves, marmalade, and jelly. Kumquats appear more commonly in the modern market as a martini garnish, replacing the classic olive. They can also be sliced and added to salads. In this research, propagation of Fortunella 